\title{
The Analysis of Publicity Slogan English Translation based on Translation Eco-environment and Three-dimensional Principle Perspective
}

\author{
Xie Shuiying
}

\author{
Guangxi Vocational and Technical College
}

\begin{abstract}
Keywords: Translation Eco-environment; Three-dimensional principle perspective; publicity slogan; English translation
\end{abstract}

\begin{abstract}
At present, ecological translation has got widespread attention at home and abroad. The article analyzes publicity slogan English translation based on translation Eco-environment and three-dimensional principles perspective to get rid of the limitation of traditional translation, innovation theory and method and make it as a guide.
\end{abstract}

Translation from the perspective of ecology has been thriving [1]. After the success of Olympic Games in 08, the high-profile World Expo was held in Shanghai. The theme of Shanghai World Expo was "Better city, better life". The exhibitors from all over the world were involved in building and participation and discussed city problems with Chinese people. While the city brings us beauty, at the same time, let us share this nice to others. Publicity slogan is a big attraction in the Expo, which not only transmits concepts, but also explains common human sense. Therefore, publicity slogan translation is important. Affected by functional translation theory, translation effect and meaning are attached great importance and care little about translation form. This is a very suitable method for slogan translation. Some scholars point out that in order to show the slogan's "call function" and "expression function", the form and effect of translation should be attached importance to. Publicity translation can be divided into two types: style driven and non-style driven. This kind of translation strategy is clearer. Take 2008 Olympic Games and 2010 Expo slogans as study object, and analyze publicity slogans English translation based on translation Eco-environment and three-dimensional principles perspective so as to make a reference for later translation.

\section{TRADITIONAL TRANSLATION PUTS EMPHASIS ON EFFECT AND NEGLECTS FORMS}

Slogan reflects people's all kinds of activities, such as military activities, business activities and political activities, and expresses the goals. There are some words and phrases as far as possible to attract readers' attention, and the external forms are popular with the observers, which has played a prominent effect of rendering effect. They have the super attraction in this case. German scholar Reiss said: "Call function also is the appeal of the text. Whether the text translation can achieve success and want to be seen again, in information transmission, infection text transfers the nonverbal purpose, and the information content is not dominated but just a second fiddle". According to this, what is important is to create artistic conception and effect in translation, however, neglect translation of communication form. When translating publicity slogans, the use of this strategy is very good, and it is a kind of effective translation means.

In fact, Reiss translates according to the effect so as to realize the infection of the text. This method can also help the translators no longer restricted to the form and structure of the text, and shift the focus of the translation to information expression. However, for the translation stresses the artistic conception form, I personally think is a wrong way. In fact, as mentioned by Reiss, compared to meaning expression, language form is not in a major position, such as domestic social education slogan, economic slogan and cultural governance slogan. The emphasis on family planning work is single-minded, which is the necessary guarantee of the country's rich and strong, so you have to practice family planning. "Mother controls, whole family's honor". From semantics 
angle, this kind of slogan can be translated word by word, but the received effect may not be ideal because this kind of slogan not only pays attention to the external form, also reflects distinctive linguistic features at the same time. In the very great degree, it is different from everyday language. With Era development, slogan form also presents diversified development trend. A lot of slogans are no longer the past "marketing" form, and turn to express tilt. Emphasize on the effective combination of content and target, which requires translators to follow diversification. Reiss' practice of translation is inappropriate, so the adjustment is necessary.

\section{OTHER FORMS OF SLOGAN TRANSLATION STRATEGIES}

Traditional translation focuses on form and effect and lacks translation strategies. Author Li Dechao and Wang Kefei put forward their own translation strategies, namely mentioned in the article "driver and non-driver's slogans". Translation cannot leave publicity slogans and determine how to use translation strategies, such as literal translation, translation modify or supplement. The two scholars think that before slogan translation, understand the slogan's express information and late anticipation, carefully analyze slogan drive used by slogan form, or use non-driving slogan. So, specifically analyze the features of the text, and investigate the characteristics. Determine the translation article, if necessary, compensate the corresponding text. This is a very good translation strategy. This paper analyzes publicity slogan English translation based on translation Eco-environment and three-dimensional principles perspective.

\section{SLOGAN TRANSLATION BASED ON ECO-ENVIRONMENT PERSPECTIVE}

Ecological environment is an important concept in the theory of ecological translation. Chinese famous scholar $\mathrm{Hu}$ Geng first presented it. It is a new type translation theory. From ecological point of view, it is also a kind of translation studies. The research is based on the integration of ecology, uses the perspective of translation and narrative method, and studies the nature, standards and methods of translation and imagines performance. According to the concept of ecological scholars, translation is a kind of ecological environment adaption. There are two aspects in translation which cannot be ignored: translator's ecological environment and translator's right choice. Translation environment embodies in the translation's original environment, the original language and translated language environment, which contains language communication activities of the translator, the translator, the cultural level of the translator, the original author and the readers, the interacting group. The translation of ecological environment is a collection of a variety of factors, which directly restricts translation. The publicity slogans in Beijing Olympic Games and Shanghai World Expo are an example. This paper puts forward a personal opinion as a reference application.

At present, the basic theory of ecological translation studies has been regarded as a prototype. This theory has extensive application in in literary translation, public signs translation and translation studies [2]. "One world, one dream" is the publicity slogan used in Beijing Olympic Games. This slogan is the embodiment of Olympic spirit, and also values expression. Its conceivable popularity is high. Olympic committee also praised it. In addition, this slogan has prominent structure features: the two "ones" are a sharp contrast of beauty in the slogan. "Word" and "dream" mutually set off, and this slogan give a person with great shock both in sound and rhythm, which is concise and lively and has profound significance. But some scholars believe that a world seems to be inappropriate, because the word has wide application range. Therefore, certain space intertextuality is conceivable, however, the translation is not a world's meaning.

At present, the principle and method of loanword translation are carried out extensive research, including a lot of analysis, such as speech, form, meaning and the nature of Chinese characters [3]. Scholar Wang Dongfeng points out that this not only expresses the translators' revealing things, but also contains more than one symbolic expression. From the west usage "one word", we find that the word is more controversial, especially in political philosophy. People use the word in derogatory sense more, and from most netizens' point of view, this change is single. It also indicates that "one world" is not welcomed in the west. In addition, the word and hegemonism in the United States 
have inevitable connection. Beijing Olympic Games is not very consistent to use this word. Personally, I think this view is not reasonable. "One world, one dream", so, from the context level it is feasible, but each decomposition is not desirable. Second, "a world" is with strong political color. The slogan's original meaning is (" one world, one hope "), which has not quit the historical stage. As "a world, a hope", one of the world AIDS day slogans, if we study the publicity slogan from the perspective of ecological translation, a specific language environment is also formed. Maybe the problem can be solved.

\section{TRANSLATION BASED ON THREE DIMENSIONAL PRINCIPLE PERSPECTIVE}

Ecological environment is one aspect and the essence of translation is also an aspect. The process of translation needs a new translation strategy and insists on translation principle, translation criteria. Based on publicity slogan, from stereo visual angle, namely the angle of culture, communication and language, realize the translation of the original. Translators need to do the corresponding translation from a multidimensional perspective. Ecological translation theory, translation process and translator lack necessary language environment, and need to use the language more than three dimensions. There could be a proper translation. Personally, in Shanghai World Expo, the volunteers had their own publicity slogans, such as "lovelier city". This translation used three different kinds of language forms, which are three dimensional transformations.

In adaptive choice of language, request the translator according to the different language form make the corresponding transformation while translating the original. The language dimension is conducted at different level. Analysis of English and Chinese two languages, as is often the case, English language is seen as a "hypotaxis" language, which needs the effective help of each language ingredients to understand the meaning and expression. Chinese is understood as a "parataxis" language, in other words, to understand and express it, we need each sentence's consciousness and speculation, such as "our city, your joy" in English. It is the embodiment of multiple "parataxis". Using the equivalent length of the two words "our city" and "your joy", including the same number of words, syllables, in stark contrast, however, this does not represent all. With "city" and "joy", it will also bring cross-eyed implication, which can be said to express the meaning of language cleverly, and realize ingenious transformation of different language dimension.

Culture's adaptability choice is the translator's interpretation of two kinds of language cultural connotations. The adaptation choice is based on original and translation by comparing the difference between the contents at the same time. Without from the perspective of the target language, this will cause the deviation of understanding of the content. Translators should focus on two languages' significant differences in the process of language translation. For example, Han culture advocates more strength with more people, and we need to create value for society and serve the society in time. When translators are on a language conversion, they cannot ignore the culture behind the language system. British and American culture attaches importance to the role of individuals. Their own existences value significance. Shanghai World Expo publicity slogan, "With me, the city is more lovely", just embodies the calling function, and the purpose is to let people better put into service for Shanghai World Expo in Shanghai, and contribute to the success of it. The English translation version is "our city, you joy". This is to make foreign friends feel at home and is a kind of respect to readers. Chinese forms of publicity slogan and English forms of publicity slogan embody different language dimension conversion.

Accommodation is the translation of ecological environment, which conforms to the typical elements in the translation, and translation is to keep up with the typical elements of the translator and the target language [4]. Corresponding transformation of communicative dimensions requires translators to carry on the corresponding transformation in different communication environment to adapt to the social environment. In front of the adaption of communication dimensions, the translator needs not only convert information into another form, but also realize the transition of different cultural connotations. It is also a key communication, and the translator should pay attention to original intention of language translation and different language types' propaganda and accept object differences. China's "With me, the city is lovelier" object is Shanghai citizen in 
Shanghai World Expo, who are committed to serve Shanghai World Expo and show their enthusiasm. English "Our city, your joy" object is the native speaker of English. Welcome visitors from afar show the beauty of Shanghai and the Expo. Communication object is changed. The dimension of communication is the premise of communication, and the difference between Chinese and English is effective transformation verification.

\section{CONCLUSION}

Publicity slogans' English translation requires the translators grasp the effective translation strategies based on ecological environment and three dimensional principle perspectives. Seen from the above analysis, in slogan translation, translators combine with "three dimensional" principle to make proper choice in specific translation environment. In this way, some of the problems in English translation can be easily solved, moreover, it also illustrates that translation eco-environment and three-dimensional principles perspective play a key role in English slogan translation. It can offer reference in publicity slogans in English translation for research workers engaging in it.

\section{REFERENCE}

[1] Hu Gengshen. Ecological translation's "vision" and "new"----Comparative study and relevant questions reply of different translation approach [J]. Journal of Foreign Languages in China, 2014, (5): 104-111.

[2] Ding Shilei. Theory of translation ecology environment [D]. Anhui University, 2013.

[3] Zhou Zhijun. English source loanwords translation from the perspective of ecology [D]. Central South University, 2013.

[4] Huo Xing. Tourist attractions signs translation from Chinese into English study from Ecological translation view [D]. Yunnan Normal University, 2014. 